\title{
How many RPAS can be safely integrated in non- segregated airspace?
}

\author{
Javier A. Pérez-Castán ${ }^{* 1}$, Fernando Gómez Comendador ${ }^{1}$, Alvaro Rodriguez-Sanz ${ }^{1}$, Rosa M. \\ Arnaldo $^{1}$ and Jaime Torrecilla ${ }^{2}$ \\ ${ }^{1}$ Universidad Politécnica de Madrid, 28040, Plaza Cardenal Cisneros s/n, Madrid, Spain \\ ${ }^{2}$ ISDEFE, Calle Beatriz de Boadilla, 28040, Madrid, Spain.
}

\begin{abstract}
The forthcoming integration of Remotely Piloted Aircraft System (RPAS) is one of the cmost omplex challenges for aviation. Europe draws to allow operating RPAS and conventional aircraft in non-segregated airspace by 2025 , but this demanding perspective entails a thorough analysis of the different aspects involved. The RPAS integration in nonsegregated airspace cannot imply an increase in the safety levels. This paper assesses how the RPAS integration affects safety levels. The goal is to regulate the number of RPAS that can jointly operate with conventional aircraft regarding conflict risk. This approach benchmarks a Calculated Level of Safety (CLS) with a Target Level of Safety (TLS). Monte Carlo (MC) simulations quantify the TLS based on schedules of conventional aircraft. Then, different combinations of conventional aircraft and RPAS provide different CLS. MC simulations are performed based on probabilistic distributions of aircraft performances, entry times and geographical distribution of aircraft. The safety levels are based on a conflict-risk model because the primary metrics are average number of conflicts and average conflict duration. The methodology is applied to one flight level of en-route airspace. The results provide restrictions to the number of RPAS that can jointly operate with conventional aircraft. Particularly, the TLS is quantified for four conventional aircraft and MC simulations provide the combinations of conventional aircraft and RPAS that fulfil the CLS. The same number of RPAS than conventional aircraft shows an increase over $90 \%$ average number of conflicts and $300 \%$ average conflict time.
\end{abstract}

\section{Introduction}

The integration of Remotely Piloted Aircraft System (RPAS) in non-segregated airspace is one of the most complex and demanding challenges for the aviation community in the years ahead. The beginning of RPAS integration in non-segregated airspace is expected to be reached to the time frame 2025, according to European RPAS Steering Group [1]. This aim requires a broad and structured analysis of the current situation as well as the potential solutions to be implemented. In this way, the development of a risk-based framework to ensure the safe integration of RPAS is crucial for its achievement.

RPAS operation in upper airspace does not require higher technological developments, but it demands detailed analysis about the safety of their integration with conventional aircraft. European Aviation Safety Agency (EASA) and Federal Aviation Administration (FAA) demand that the integration of RPAS must not imply a diminish on current safety levels $[2,3]$. "Integration" refers to a future when RPAS may be expected to enter the airspace routinely without requiring special provisions [4]. A new framework will be compulsory in the future to take the operational features of RPAS into account. One of the goals of this 
framework is to allow for setting out the safety of the RPAS operation jointly with conventional aircraft [5-7].

Could RPAS fly safely in non-segregated aircraft? The complexity of the answer does not fall in, whether yes or not, because it must be yes, but the issue is about how. Currently, conventional aircraft fly according to prefixed routes that are modelled according to air traffic flow patterns, although there are several airspaces based on free-route [8]. Then, RPAS must adapt to the current airway network and current air traffic patterns. One of the main concerns is that the operational patterns of RPAS can differ from conventional aircraft ones $[9,10]$. Although RPAS could be assumed to be modelled as slow conventional aircraft, there are uncertainties about communications, navigation and surveillance issues that must be analysed in advance [11]. Due to this lack of operational and technical knowledge about RPAS, regulators and Airspace Navigation Service Providers (ANSPs) seek to introduce RPAS based on a minimum interaction with conventional aircraft $[12,13]$. The problem arises when both airspace users operate jointly in the same scenario where the interaction between them cannot be avoided.

The most complex assessments about RPAS integration have tackled three research lines. The first deals with the global problem of risk management. Clothier et al. [14] developed a framework for structuring the safety case of the RPAS operation. Moreover, various regulators assessed the main difficulties that must be solved before RPAS operation $[15,16]$. Second group analyses the risk imposed by the single flight for one RPAS in terms of the number of casualties. Several authors developed different risk models to calculate what kind of populated areas are riskier for on-ground pedestrians [17-19]. Third research line involves the development of collision/conflict-risk models for the integration of RPAS. There are several studies about RPAS collision avoidance [10,20,21] (similar to conventional aircraft situations) but few of them focus on conflict risk [22,23]. Conflict risk is a prior indicator of collision risk. However, none of those studies responds about the number of RPAS that can be safely integrated in non-segregated airspace.

With the goal of responding to the above research question, it is required to assess the safety level of the airspace and to develop one specific methodology. Manual 9689 of International Civil Aviation Organization (ICAO) [24] set out that airspace planning required a thorough analysis of every factor that could affect safety. In [25,26], authors claimed the need for airspace design fulfilling levels of safety under different operational features. Different models were developed to evaluate the collision risk based on airspace geometry [27,28]. A step further, Netjasov [29] developed a conflict-risk model to assess the level of safety, including air traffic flows. However, those studies could not model the influence of different variables as conflict geometry, density or airway length, among others. The issue underlies if we try to evaluate the airspace risk level, crucial information will be missed to understand the operational frame.

Therefore, the main goal of this research is to develop a methodology to quantify the number of RPAS that can be safely integrated in non-segregated airspace. A further goal is to analyse how the integration of RPAS impact on different safety metrics. The rest of the article is structured as follows. Section 2 presents the structure of the methodology and defines the different types of variables and indicators that must be considered. The safety metrics constitute the main outputs of the methodology to assess the viability of RPAS integration. Section 3 describes Monte Carlo (MC) simulations based on real airspace and the characterisation of the different variables. Section 4 provides and discusses the main results of the simulations by the integration of RPAS jointly with conventional aircraft. Lastly, Section 5 summarises the main contributions and further works.

\section{Methodology}


This section describes the methodology followed to tackle the problem described above based on the different elements that compose the methodology:

\subsection{Initial Conditions}

There are different variables that are fixed and required to perform the simulations. These variables constitute the initial conditions and vary for each simulation. They are basically related to air traffic features:

- Aircraft schedule. It provides the number of conventional aircraft and RPAS that are involved in each simulation. Air traffic flows can follow the operational distribution through the airspace or can be randomly simulated.

- Airways. They indicate the path that will be operated by an aircraft. The existence of airways implies that free-route airspace is not considered herein.

- $\quad$ Flight Level. It indicates the Flight Level (FL) for each aircraft through the airspace. In this study climbing or descending aircraft are not considered.

- Velocity: It indicates the ground speed for each aircraft through the airspace. It is assumed that aircraft fly at a constant speed through the airspace. Nonetheless, each aircraft flies at different velocities, then, a probabilistic distribution is considered based on statistical analyses.

- Entry time: It indicates the time at which the aircraft pierces into the airspace. Once the aircraft enters into the airspace, the aircraft flies a specific airway characterised by the velocity and FL selected. Similar to velocity, the entry time will be modelled based on a probabilistic distribution.

\subsection{Boundary Conditions}

The boundary conditions are characterised by the airspace morphology.

- Airspace geometry. The airspace geometry depends on the airspace sector characterised. It is required to select the vertical and lateral limits.

- Airways. Each airspace is constituted by fixed airways. They are characterised by their length and their spatial distribution

- Crossing points. They are the intersections between at least two airways. They are characterised by the number of airways and their crossing angles

- Crossing angle. They are the angles constituted by each airway pair. The crossing angles are crucial for the calculation of different operational factors as the critical section.

\subsection{RIT tool}

RPAS Integration Tool (RIT) is a MatLab® code developed specifically to simulate and evaluate the integration of RPAS developed by UPM [23,30]. The RIT tool allows modelling different airspaces based on above conditions. Therefore, it provides a framework to analyse the risk by the integration of RPAS. The process followed by the RIT tool is summarised in Fig 1.

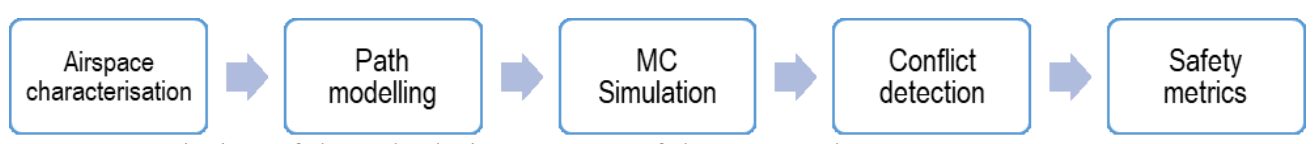

Fig. 1. Description of the calculation process of the RIT tool. 


\subsection{Safety Metrics}

For each simulation, a specific set of safety metrics are required to evaluate the safety levels for the integration of RPAS. In this work, two safety metrics are considered:

- Average number of conflicts $\left(\overline{N_{c}}\right)$. this safety metric refers to the number of times a separation minima violation can occur for each simulation, based on the aircraft schedule considered. This metric is overly extended in the literature. This is a paramount factor because it provides a quantification of the Target Level of Safety (TLS) based on the number of conflicts.

- Average conflict duration $\left(\overline{\tau_{c o n}}\right)$. This is a new safety metric that analyses the average time of the conflicts that appear for each simulation. The reason to evaluate this safety metric is to appraise if the integration of RPAS introduces modifications to the conflicts due to their low speed.

\section{Monte Carlo Simulations}

The goal of this work is to evaluate how the integration of RPAS affects above safety metrics to quantify the number of RPAS that can be safely integrated. Zaragoza airspace (LECMZGZ) in Spain is the airspace selected to quantify the TLS based on the current operation.

\subsection{Scenario description}

We have selected the Spanish airspace volume of Zaragoza "LECMZGZ". This airspace is located in the north of Spain and encompasses both lower and upper airspace, from $1000 \mathrm{ft}$ to FL 460. This airspace is characterised by high rate of en-route flights and scarce influence of climbing/descending flights. Its influence represents less than $15 \%$ of conventional aircraft. Fig. 2 shows LECMZGZ geometry, which is constituted by 14 airways and 30 crossing points.

The North-South (37.7\%) and East-West (39.5\%) corridors dominate air traffic flows of LECMZGZ airspace. Besides, LECMZGZ airspace presents an advantage because only two airways intersect at the majority of the crossing points. Particularly, this study only considered the FL 270 that was detected as the most convenient for the introduction of RPAS in previous work [23].

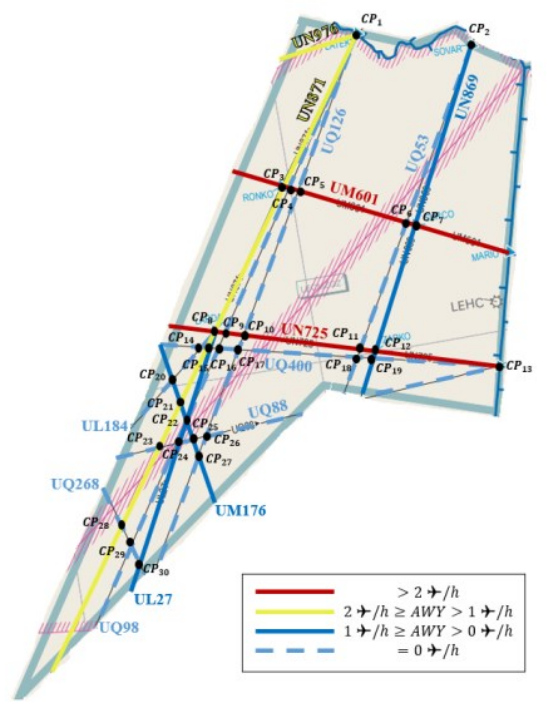


Fig. 2. Scheme of LECMZGZ airspace.

\subsection{Characterisation of MC simulations}

MC simulations were validated by carrying out different experiments. One experiment is characterised by the overall set of conventional aircraft and RPAS considered. The entry time, airway and velocity follow different probabilistic distributions based on real operational data. Then, the range of each variable varies as indicated in Table 1.

Table 1. Values of the variables for MC simulations.

\begin{tabular}{|c|c|c|}
\hline Control variables & Range & Randomness \\
\hline No. Total aircraft & $2-8$ & No \\
\hline Airway & $1-14$ (LECMZGZ) & Yes \\
\hline Entry time & $\mathrm{U}(1,3600)$ seconds & Yes \\
\hline Speed & $N_{\text {conv }}(450,20) ; N_{\text {RPAS }}(250,5)$ knots & Yes \\
\hline FL & 270 & No \\
\hline
\end{tabular}

The main characteristics of the MC simulations were:

- The TLS is calculated for the experiment of four conventional aircraft and zero RPAS because it was the maximum number of aircraft in the FL 270.

- MC simulations evaluate every feasible scenario for schedules from four conventional aircraft to four RPAS, being two the minimum number of aircraft.

- For each experiment, $10000 \mathrm{MC}$ simulations were performed to statistically obtain valid values. For each of these simulations, the Calculated Level of Safety (CLS) is calculated.

- Each aircraft operates randomly an airway of the airspace. In this study, air traffic flows distribution is not considered.

- The entry time for each aircraft is randomly defined for a period of one hour. Then, each aircraft pierces into the airspace from 0 to 36000 seconds.

- Velocity also follows a probabilistic distribution depending on the aircraft type. Based on statistical data from conventional aircraft, a normal distribution is assumed for the conventional aircraft. As no previous information of RPAS can be extracted from statistical data, it was assumed a normal distribution with a standard deviation of five knots (kts) based on data from BADA [31]. In this way, each conventional aircraft or RPAS flies with a different velocity.

- Finally, simulations only considered one FL and it is expected to increase the number of FLs and interaction between aircraft in further work.

\section{Discussion of results}

The final goal of this work is to quantify the number of RPAS that can be safely integrated in non-segregated airspace. The TLS is calculated for four conventional aircraft, which is the current rush hour, and compared with the CLS for each experiment, combinations of conventional aircraft and RPAS.

\subsection{Average number of conflicts $\left(\overline{N_{c}}\right)$}

The first safety metric is the number of times the separation minima are violated between aircraft pairs. The number of conflicts was calculated for MC simulations of the different experiments. For each one of 16 experiments of conventional aircraft and PRAS, 10000 MC simulations were performed varying the initial variables. 
Table 2 provides the average number of conflict detected for each simulation $\left(\overline{N_{c}}=N_{c} / 10000\right)$. In red the experiments that exceed the TLS are highlighted in red and in yellow one special experiment (1 conventional aircraft and 2 RPAS).

Table 2. $\overline{N_{c}}$ for each experiment.

\begin{tabular}{|c|c|c|c|c|c|}
\hline \multirow{2}{*}{ No. Conv } & \multicolumn{5}{|c|}{ No. RPAS } \\
\cline { 2 - 6 } & $\mathbf{0}$ & $\mathbf{1}$ & $\mathbf{2}$ & $\mathbf{3}$ & $\mathbf{4}$ \\
\hline 0 & 0 & 0 & 0.029 & 0.090 & 0.183 \\
\hline 1 & 0 & 0.046 & 0.116 & 0.228 & \\
\hline 2 & 0.018 & 0.109 & 0.228 & & \\
\hline 3 & 0.054 & 0.185 & & & \\
\hline 4 & 0.115 & & & & \\
\hline
\end{tabular}

The primary outcomes are:

- The increase of the number of aircraft (both conventional aircraft of RPAS) entails the increase of conflicts detected.

- The comparison between the same number of conventional aircraft and RPAS provides greater results for the RPAS. For instance, three conventional aircraft provide 0.054 conflicts per simulation while three RPAS provide 0.09 (90\% increase).

- In the same way, experiments with the same total number of aircraft (RPAS and conventional aircraft combinations) provide riskier values in the case RPAS dominate conventional aircraft.

- Moreover, if Table 2 is analysed following the diagonals (the total number of aircraft remains constant), the number of conflicts detected evolved following a parabolic function, see Fig. 3.

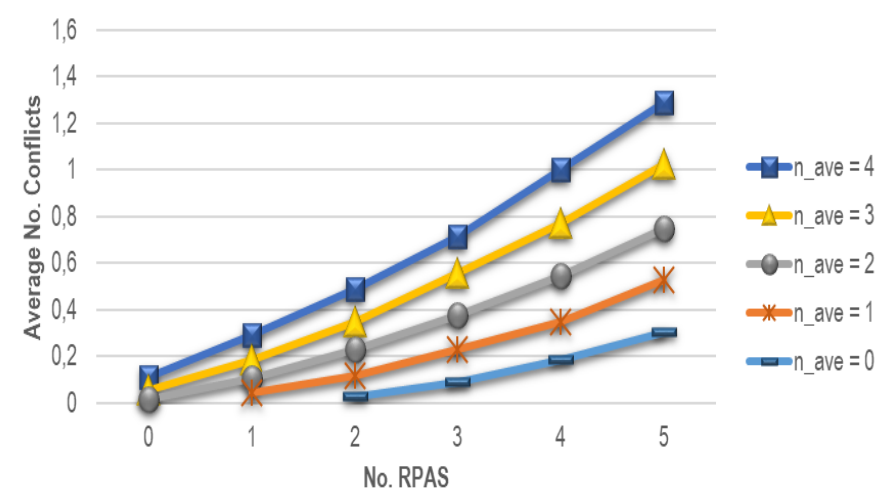

Fig. 3. Evolution of $\overline{N_{c}}$ for each experiment.

- The last analysis compares the experiments with the same number of RPAS than conventional aircraft. As commented before, the average number of conflict increase for the cases with RPAS. However, the evolution differs from was expected because the larger values were obtained for intermediate combinations of RPAS and conventional aircraft, see Fig. 4. 


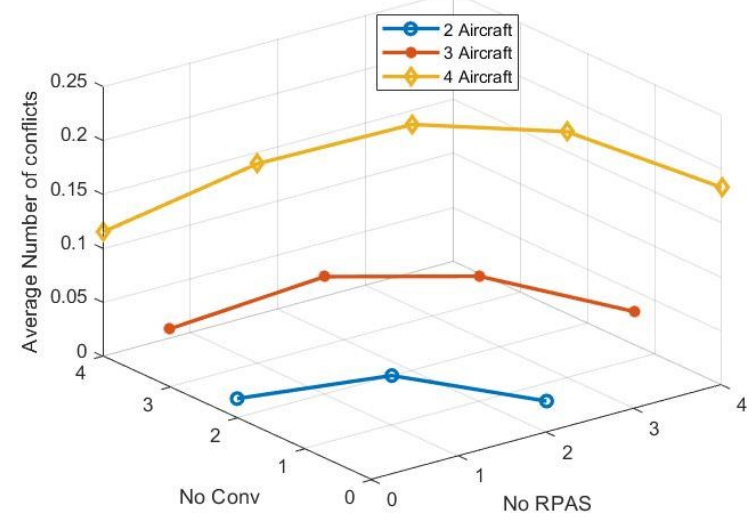

Fig. 4. Evolution of $\overline{N_{c}}$ for combinations of the same number of aircraft.

Lastly, there are six configurations allowing the integration of RPAS with conventional aircraft in LECMZGZ at FL 270. All of them involve three or less aircraft. Therefore, the integration of RPAS implies that the number of aircraft must be reduced by one $(25 \%$ decrease) to ensure current safety limits.

\subsection{Average conflict duration $\left(\overline{\tau_{\text {con }}}\right)$}

In this work, a new safety metric is considered to analyse how varies the average conflict duration by the introduction of RPAS. For each conflict, its duration is calculated for every experiment and simulation. Table 3 provides the values calculated for each experiment. In red the experiments that exceed the TLS are highlighted in red and in yellow one special experiment (1 conventional aircraft and 2 RPAS).

Table 3. $\overline{\tau_{\text {con }}}$ (s) for each experiment.

\begin{tabular}{|c|c|c|c|c|c|}
\hline \multirow{2}{*}{ No. Conv } & \multicolumn{5}{|c|}{ No. RPAS } \\
\cline { 2 - 6 } & $\mathbf{0}$ & $\mathbf{1}$ & $\mathbf{2}$ & $\mathbf{3}$ & $\mathbf{4}$ \\
\hline $\mathbf{0}$ & 0 & 0 & 7.66 & 25.78 & 51.57 \\
\hline $\mathbf{1}$ & 0 & 4.78 & 17.40 & 41.99 & \\
\hline $\mathbf{2}$ & 2.37 & 12.51 & 30.14 & & \\
\hline $\mathbf{3}$ & 8.23 & 21.51 & & & \\
\hline $\mathbf{4}$ & 17.26 & & & & \\
\hline
\end{tabular}

The primary outcomes are similar to the previous safety metric:

- The introduction of RPAS increases the value of this safety metric and provides similar results than the average number of conflicts. However, there are some experiments that increase the limit value of the experiment of four conventional aircraft. They were the experiment of one conventional aircraft and two RPAS and zero conventional aircraft and three RPAS. Both cases were valid experiments in the previous indicator but the average conflict duration did not fulfil this safety metric. Although the combination of one conventional aircraft and two RPAS can be accepted because the increase is reduced, the experiment with only three RPAS cannot be accepted.

- The average conflict duration is larger for the experiments with only RPAS but with higher ratios than the average number of conflicts $(300 \%$ instead of $90 \%)$. It is obvious that the lower speed of the RPAS entails the increase of the conflict duration. This is a 
critical issue to bear in mind because the conflict and risk severity increase with the use of RPAS instead of conventional aircraft. However, in the experiments with a combination of conventional aircraft and RPAS the average conflict duration increases until $200 \%$. Table 4 provides the values for the different combinations.

Table 4. $\overline{\tau_{\text {con }}}$ ratio for the same number of aircraft.

\begin{tabular}{|c|c|c|c|}
\hline No. Conv & No. RPAS & $\overline{\boldsymbol{\tau}_{\text {con }}}(\mathbf{s})$ & Ratio between $\overline{\boldsymbol{\tau}_{\text {con }}}(\mathbf{s})$ \\
\hline 2 & 0 & 2.37 & \multirow{2}{*}{3.23} \\
\hline 0 & 2 & 7.66 & \multirow{2}{*}{3.13} \\
\hline 3 & 0 & 8.23 & \multirow{2}{*}{2.99} \\
\hline 0 & 3 & 25.74 & \multirow{2}{*}{5.28} \\
\hline 4 & 0 & 17.26 & \multirow{2}{*}{2.61} \\
\hline 0 & 4 & 51.57 & \multirow{2}{*}{2.07} \\
\hline 2 & 0 & 2.37 & \\
\hline 2 & 1 & 12.51 & \\
\hline 3 & 0 & 8.23 & \multirow{2}{*}{} \\
\hline 3 & 1 & 21.51 & \\
\hline 4 & 0 & 17.26 & \multirow{2}{*}{} \\
\hline 4 & 1 & 35.65 & \\
\hline
\end{tabular}

- The integration of RPAS entails a limitation to the total number of aircraft. The introduction of conventional aircraft provides better results than the introduction of RPAS, as was expected. To increase the number of conventional aircraft from two to three implies an increase of $300 \%$ while the introduction of one RPAS increased by $500 \%$ this value.

- In this case, the total number of RPAS that can be integrated into non-segregated airspace decreased by one and two depending on the combinations of aircraft. RPAS are slower than conventional aircraft, which implies they are more time exposed to conflict.

Therefore, the total number of RPAS that can be safely integrated jointly with conventional aircraft is two in the case no conventional aircraft operates (an air traffic reduction of $50 \%$ with respect to current TLS). However, combinations of RPAS and conventional aircraft allow the introduction of three aircraft (an air traffic reduction of $25 \%$ with respect to current TLS). Hence, the average conflict duration was the most restrictive safety metric for the introduction of RPAS because it blocked the combination of three RPAS without conventional aircraft.

\section{Conclusions}

This research developed a methodology to quantify the number of RPAS that can be safely integrated in non-segregated airspace. The methodology fixed a TLS depending on different safety metrics underlined by the current operation of conventional aircraft. Safety metrics selected were the average number of conflict and the average conflict duration for each simulation. The statistical validation of the simulations were performed based on Monte Carlo simulations. MC simulations allowed modifying the operational variables (air traffic information) of the aircraft involved - such as entry time, velocity and airway. Based on current operation in the airspace, different TLS were obtained for two safety metrics. Consequently, MC simulations analysed different experiments where the combination of RPAS and conventional aircraft varied from zero to four aircraft. This methodology was applied to the Spanish airspace LECMGZ and the FL 270, simulating random air traffic distributions. The TLS of LECMZGZ was calculated for four conventional aircraft in the rush hour. The maximum number of RPAS without conventional aircraft was limited to two 
(50\% decrease) and combinations of RPAS and conventional aircraft was limited to three ( $25 \%$ decrease). The safety metric most critical was the average conflict duration due to the effect of the lower RPAS velocity. Average number of conflicts allowed the introduction of three RPAS without conventional aircraft but average conflict duration did not allowed. Further research lines must focus on the calculation of the safety metrics for different airspaces bearing in mind the real air traffic distributions. Moreover, the introduction of climbing or descending aircraft could be crucial to correctly quantify TLS with new restrictions for the integration of RPAS.

\section{Acknowledgements}

This Project has been developed under the OIDATM (Observatory for the Advancement of Air Traffic Management) promoted by ISDEFE. Particularly, the authors would like to acknowledge Gonzalo Águeda and Cristina Altemir from UPM and Miguel A. Martín Blanco, Maria Anta Garcia and Susana Duran Vizuete from ISDEFE.

\section{Conflict of interest}

The authors declare no conflict of interest.

\section{References}

1. European RPAS Steering Group. Roadmap for the Integration of Civil Remotely Piloted Aircraft Systems into the European Aviation System. 2013.

2. EASA. Advance NPA 2015-10: Introduction of a Regulatory Framework for the Operation of Drones. 2015.

3. FAA. 8130.34D - Airworthiness Certification of Unmanned Aircraft Systems and Optionally Piloted Aircraft. 2017.

4. $\quad$ SESAR. “European ATM Master Plan 2015.” 2015 p. 140. doi:10.2829/240873.

5. R.R. Cordón,F. Javier,S. Nieto. RPAS Integration in Non-Segregated Airspace : The SESAR Approach System Interfaces Needed for Integration. 2014.

6. ICAO. Manual on Remotely Piloted Aircraft Systems (Rpas). 2015. 2015.

7. $\quad$ SESAR. Modernising the European Sky. 2014.

8. E. Calvo-Fernández,L. Perez-Sanz,J.M. Cordero-García,R.M. Arnaldo-Valdés. "Conflict-Free Trajectory Planning Based on a Data-Driven Conflict-Resolution Model.” J. Guid. Control. Dyn. 4032017 pp. 615-627. doi:10.2514/1.G000691.

9. J.A. Pérez-Castán,F. Gómez Comendador,R.M. Arnaldo-Valdés,L. Hernández Gila,J. Torrecilla Puebla. Conflict-Resolution Algorithms for Separation Minima Definition of Rpas in a Non-Segregated Airspace. 2017.

10. P. Pierpaoli,A. Rahmani. "UAV Collision Avoidance Exploitation for Noncooperative Trajectory Modification.” Aerosp. Sci. Technol. 12017 pp. 1-11. doi:10.1016/j.ast.2017.12.008.

11. J. Bueno,C. Regidor,D. Escribano,F. Ferrández,M. Vega. Human and Technical Performance Aspects in RPAS Integration Trials in Controlled Airspace. 2016.

12. C. Allignol,N. Barnier,N. Durand. Detect \& Avoid, UAV Integration in the Lower Airspace Traffic. 2016.

13. R.A. Clothier,D.A. Greer,D.G. Greer,A.M. Mehta. "Risk Perception and the Public Acceptance of Drones." Risk Anal. 3562015 pp. 1167-1183. doi:10.1111/risa.12330.

14. R.A. Clothier,B.P. Williams,N.L. Fulton. "Structuring the Safety Case for Unmanned Aircraft System Operations in Non-Segregated Airspace.” Saf. Sci. 79 
2015 pp. 213-228. doi:10.1016/j.ssci.2015.06.007.

15. R.A. Clothier,J.L. Palmer,R.A. Walker,N.L. Fulton. "Definition of an Airworthiness Certification Framework for Civil Unmanned Aircraft Systems." Saf. Sci. 4962011 pp. 871-885. doi:10.1016/j.ssci.2011.02.004.

16. K. Dalamagkidis,K.P. Valavanis,L.A. Piegl. “On Unmanned Aircraft Systems Issues, Challenges and Operational Restrictions Preventing Integration into the National Airspace System.” Prog. Aerosp. Sci. 44 7-8 2008 pp. 503-519. doi:10.1016/j.paerosci.2008.08.001.

17. Civil Aviation Safety Authority,Australian Government. Review of Aviation Safety Regulation of Remotely Piloted Aircraft Systems. 2018.

18. R. Clothier,R. Walker,N. Fulton,D. Campbell. A Casualty Risk Analysis for Unmanned Aerial System (UAS) Operations over Inhabited Areas. 2007.

19. R. Melnyk,D. Schrage,V. Volovoi,H. Jimenez. “A Third-Party Casualty Risk Model for Unmanned Aircraft System Operations.” Reliab. Eng. Syst. Saf. 124 2014 pp. 105-116. doi:10.1016/j.ress.2013.11.016.

20. Y. Lin,S. Saripalli. Collision Avoidance for UAVs Using Reachable Sets. 2015.

21. A. Zarandy,T. Zsedrovits,B. Pencz,M. Nameth,B. Vanek. A Novel Algorithm for Distant Aircraft Detection. 2015.

22. C.A. Persiani,S. Bagassi. "Route Planner for Unmanned Aerial System Insertion in Civil Non-Segregated Airspace." Proc. Inst. Mech. Eng. Part G J. Aerosp. Eng. 22742013 pp. 687-702. doi:10.1177/0954410012439975.

23. J.A. Pérez-Castán,F. Gómez Comendador,A. Rodríguez-Sanz,I. Armas Cabrera,J. Torrecilla. "RPAS Conflict-Risk Assessment in Non-Segregated Airspace." Saf. Sci. 111 March 20182019 pp. 7-16. doi:10.1016/j.ssci.2018.08.018.

24. ICAO. Doc 9689-AN/953 - Manual on Airspace Planning Methodology for the Determination of Separation Minima. 1998.

25. J. Shortle,S. Noh,L. Sherry. "Collision Risk Analysis for Alternate Airspace Architectures.” AIAA/IEEE Digit. Avion. Syst. Conf. - Proc. 2017-Septe 2017. doi:10.1109/DASC.2017.8102093.

26. L.M.B.C. Campos,J.M.G. Marques. "On a Dimensionless Alternative to the ICAO Target Level of Safety.” Proc. Inst. Mech. Eng. Part G J. Aerosp. Eng. 23092016 pp. 1548-1557. doi:10.1177/0954410015621336.

27. K.E. Geisinger. “Airspace Conflict Equations.” Transp. Sci. 1921985 pp. 139153. doi:10.1287/trsc.19.2.139.

28. Siddiqee W. "A Mathematical Model for Predicting the Number of Potential Conflict Situations at Intersecting Air Routes.” Transp. Sci. 721973 pp. 571-577. doi:10.1287/trsc.7.2.158.

29. F. Netjasov. "Framework for Airspace Planning and Design Based on Conflict Risk Assessment Part 1 : Conflict Risk Assessment Model for Airspace Strategic Planning." Transp. Res. Part C Emerg. Technol. 242012 pp. 190-212. doi:10.1016/j.trc.2012.03.002.

30. J.A. Pérez-Castán,F.G. Comendador,Á. Rodriguez-Sanz,R.M. Arnaldo Valdés,G. Agueda. "RPAS Integration in Non-Segregated Airspace: Safety Metrics for Tactical Planning." Proc. Inst. Mech. Eng. Part G J. Aerosp. Eng. 002019 p. 095441001986126. doi:10.1177/0954410019861263.

31. EUROCONTROL. BADA Aircraft Performance Model. 